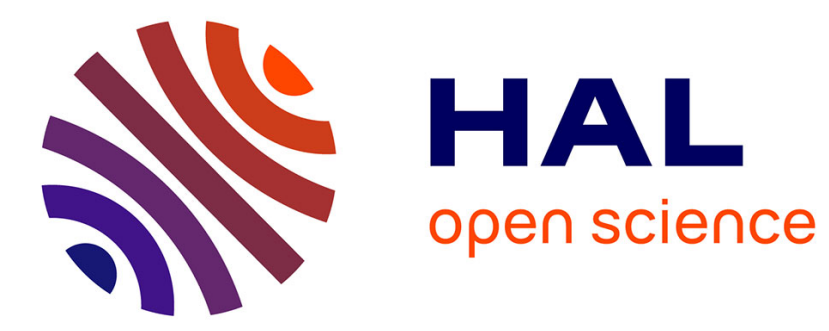

\title{
Chaotic Dynamics of Flags from Recurring Values of Flapping Moment
}

\author{
Emmanuel Virot, Davide Faranda, Xavier Amandolese, Pascal Hemon
}

\section{To cite this version:}

Emmanuel Virot, Davide Faranda, Xavier Amandolese, Pascal Hemon. Chaotic Dynamics of Flags from Recurring Values of Flapping Moment. International journal of bifurcation and chaos in applied sciences and engineering , 2017, 27 (02), pp.1750020. 10.1142/S0218127417500201 . hal-02886449

\section{HAL Id: hal-02886449 \\ https://hal.science/hal-02886449}

Submitted on 8 Jul 2020

HAL is a multi-disciplinary open access archive for the deposit and dissemination of scientific research documents, whether they are published or not. The documents may come from teaching and research institutions in France or abroad, or from public or private research centers.
L'archive ouverte pluridisciplinaire HAL, est destinée au dépôt et à la diffusion de documents scientifiques de niveau recherche, publiés ou non, émanant des établissements d'enseignement et de recherche français ou étrangers, des laboratoires publics ou privés. 


\title{
Chaotic dynamics of flags from recurring values of flapping moment
}

\author{
Emmanuel Virot \\ LadHyX, CNRS UMR 7646, École Polytechnique \\ 91128 Palaiseau, France \\ emmanuel.virot@polytechnique.edu \\ Davide Faranda \\ SPEC \& LCSE CEA Saclay \\ 91191 Gif-sur-Yvette, France \\ Xavier Amandolese \\ LadHyX, CNRS UMR 7646, École Polytechnique \\ 91128 Palaiseau, France \\ Conservatoire National des Arts et Métiers \\ 75003 Paris, France \\ Pascal Hémon \\ LadHyX, CNRS UMR 7646, École Polytechnique \\ 91128 Palaiseau, France
}

Received (to be inserted by publisher)

\begin{abstract}
The performance of recently proposed flag-based energy harvesters is strongly limited by the chaotic response of flags to strong winds. From an experimental point of view, the detection of flag chaotic dynamics were scarce, based on the flapping amplitude and the maximal Lyapunov exponent. In practice, tracking the flapping amplitude is difficult and flawed in the large oscillation limit. Also, computing the maximal Lyapunov exponent from time series of limited size requires strong assumptions on the attractor geometry, without getting insurance of their reliability. For bypassing these issues, (1) we use a time series which takes into account the whole dynamic of the flag, by using the flapping moment which integrates its displacements, and (2) we apply an algorithm of detection of chaos based on recurring values in time series.
\end{abstract}

Keywords: flag flutter, chaotic dynamic, time series

\section{Introduction}

The irregular flapping of flags is a common experience, as illustrated by physicists playing with their immediate environment in the 70's: "How far away is the nearest strange attractor? [...] That flag snapping erratically in a steady breeze?" [Gleick, 1987]. As earlier observed in wind tunnels, the flag oscillations become "violent and irregular" [Taneda, 1968] at high wind velocity. Numerically, the intuitive scenario "straight state - periodic flapping - chaotic flapping" has been stated by several numerical simulations [Yadikin et al., 2001; Connell \& Yue, 2007; Alben \& Shelley, 2008; Michelin et al., 2008; Huang \& Sung, 
2010], but scarce experimental works have undertaken to confirm it. Recent prototypes of energy harvesters based on the periodic oscillations of flags (see e.g. [Xia et al., 2015; Virot et al., 2016]) are now motivating quantitative estimations of the range of wind velocities allowing periodic motions as a function of the characteristics of flags. As we will see, this requires to tackle two major issues. Firstly, (1) the attempts at our knowledge to detect flag chaotic dynamics [Ait Abderrahmane et al., 2011; Zhao et al., 2012; Ait Abderrahmane et al., 2012] were based on the tracking of a single point of the flag with a laser beam perpendicular to the flag, reasonable in the limit of small oscillations but biased in practice since typical flapping amplitudes can be of the order of the flag length [Shelley \& Zhang, 2011]. A global observable is preferable to take into account the whole dynamics: in this paper we use the moment around the flagpole induced by fluid forces (hereafter called flapping moment). Secondly, (2) an equivalent attractor is classically reconstructed with the time-delay method [Packard et al., 1980; Takens \& Mañé, 1981] to characterize the rate of separation of close trajectories. Then, the maximal Lyapunov exponent is usually computed but requires assumptions on the attractor geometry. We suggest that a method stating its convergence is more appropriate: we use an algorithm based on recurring values in a time series (as proposed in [Faranda et al., 2012]), combining the work of Poincaré [Poincaré, 1890] and the extreme value theory [Leadbetter et al., 1982]. Unlike classical tools, the method is based on theoretical results for chaotic systems which predict the type of the underlying distribution of recurrences. This information allows to check whether the results obtained experimentally agree with the theoretically expected distribution. It allows to include statistical tests in the algorithm and increase the robustness of the results. This is a crucial issue when dealing with relatively short time series.

\section{Experimental set-up}

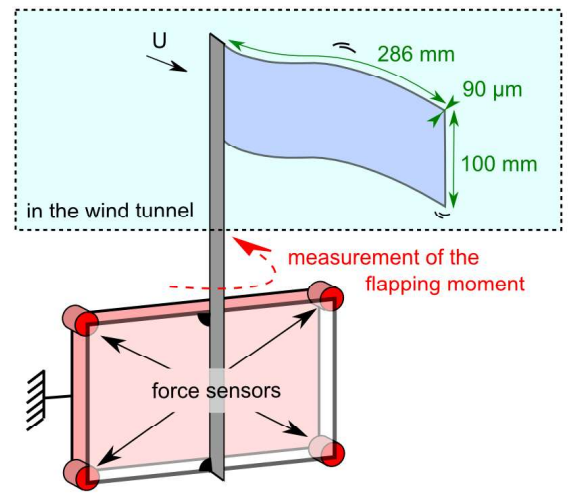

Fig. 1. Schematic representation of the experiment: the flag is placed in a wind tunnel and fixed at a flagpole which is connected to four force sensors. The flapping moment is reconstructed from the signals of the force sensors.

The flag studied is rectangular, made of bi-oriented polypropylene, with a mass density $890 \mathrm{~kg} \cdot \mathrm{m}^{-3}$ and a bending rigidity $1.3 \times 10^{-4}$ N.m (Fig. 1). Since it does not sag at rest, gravity effects are neglected. The flag is placed in an Eiffel-type wind tunnel of rectangular cross-section width $\times$ height $=260 \mathrm{~mm} \times 240 \mathrm{~mm}$ and it is clamped in a flagpole of thickness $4 \mathrm{~mm}$. The distance to the wind tunnel walls below and above the flag is respectively $40 \mathrm{~mm}$ and $100 \mathrm{~mm}$. The wind velocity can be varied up to $15 \mathrm{~m} / \mathrm{s}$ by increments of $0.4 \mathrm{~m} / \mathrm{s}$, for a Reynolds number based on the flag length lower than $3 \times 10^{5}$ and a turbulence level - the relative importance of velocity temporal fluctuations - about $0.4 \%$. The instantaneous flapping moment is reconstructed by a linear combination of the force sensor signal, as shown in Fig. 2, with 1024 acquisitions per second. The data are filtered above $60 \mathrm{~Hz}$ and the noise is reduced by treating independently six blocks of $4 \mathrm{~s}$ (with an overlap of 50\%); the resulting frequency resolution is then $0.25 \mathrm{~Hz}$ (see also [Virot et al., 2013] for further conception details). Since the acquisition lasts $14 \mathrm{~s}$, the time series contain approximately $1.4 \times 10^{4}$ observations, spanning more than hundred flapping periods in general. The length of the time series is irremediably limited, due to the experimental procedure. Indeed, a typical test 
consists of successive acquisitions, each separated by some minutes to reach steady states. Since the wind velocity range $U=0-15 \mathrm{~m} / \mathrm{s}$ is scanned with increments of $0.4 \mathrm{~m} / \mathrm{s}$, the flag undergoes about $10^{5}$ cycles, which in practice leads the flag to fracture by fatigue [Virot, 2015].

\section{Detection of chaos}

Some ideas pertaining to the extreme value theory are recalled to design the algorithm of detection of chaos (see ref. also [Leadbetter et al., 1982]). Let independent and identically distributed observations $x(t)$ be sampled into $n$ blocks, each containing $m$ observations. We know that in the limit $n, m \rightarrow \infty$ the cumulative distribution function of block maxima $\left(M_{j}\right)_{j=1, \ldots, n}$ becomes [Gnedenko et al., 1943]:

$$
F(x)=\exp \left\{-\left[1+\xi\left(\frac{x-\mu}{\sigma}\right)\right]^{-1 / \xi}\right\},
$$

where $\mu \in \mathbb{R}$ is the "location parameter", $\sigma \in \mathbb{R}^{+*}$ is the "scale parameter" and $\xi \in \mathbb{R}$ is the "tail index". The tail index indicates the thickness of the tail of the distribution: Fréchet distribution $(\xi>0)$, Gumbel distribution $(\xi=0)$ or Weibull distribution $(\xi<0)$. Originally, this theory has been devised for the study of independent and identically distributed variables $x(t)$. More recently, important contributions have been given by [Coulet, 2001; Freitas \& Freitas, 2008; Freitas et al., 2009; Gupta et al., 2011; Faranda et al., 2011] to deal with variables with a dependency structure. This is possible by adapting the Poincaré recurrence theory for dynamical systems [Poincaré, 1890] to the study of time series: if one considers a point $\zeta$ and takes as observable the function $y(t)=g(\operatorname{dist}(\zeta, x(t)))$ i.e. the time series of the distances between $\zeta$ and the other points conveniently weighted by a function $g(\cdot)$, it has been proven [Freitas et al., 2009 ] that the (block) maxima of the (sampled) observable $y(t)$ converge to the generalized extreme value distribution [eq. (1)] providing that the underlying system is chaotic (meaning intuitively that a partial loss of dependency structure is achieved). In particular, if $g(\cdot)=-\log (\cdot)$ is selected, one gets convergence towards the Gumbel distribution i.e. $\xi=0$ [Lucarini et al., 2012]. A key point is that the converse is also assumed: by taking a time series of an unknown dynamical system one can detect whether it is chaotic or not by fitting the distribution of maxima of the time series $y(t)=-\log (\operatorname{dist}(\zeta, x(t))$ to the Gumbel distribution. As a result, a successful fit expressed that the time series is chaotic and an unsuccessful fit means that it is not chaotic [Faranda et al., 2012]. In such a framework, there is no assumptions on the attractor geometry (e.g. its embedding dimension). These ideas have recently been applied to the detection of chaos in the standard map [Faranda et al., 2012].

\section{Algorithm}

Accordingly, the following algorithm is proposed:

(1) Consider a time series $x(t)$ and select an arbitrary point $\zeta$, as shown in Fig. 2(a).

(2) Compute the series $y(t)=-\log (\operatorname{dist}(\zeta, x(t)))$, where "dist" refers to the distance operator [Fig. 2(a)].

(3) Once divided the series $y(t)$ into $n$ blocks, extract the maxima $\left(M_{j}\right)_{j=1, \ldots, n}$. The maxima of $y(t)$ are related to the minima of $\operatorname{dist}(\zeta, x(t))$.

(4) Fit the maxima to the generalized extreme value distribution [eq. (1)] with an L-moments method [Faranda et al., 2012] and register the best fit parameter $\xi$ [Fig. 2(c,d)].

(5) Average the best fit tail index $\xi$ for several points $\zeta$ in order to reduce the uncertainty (we use hundred random values $\zeta$ in the following computations).

(6) Perform a Lilliefors test [Lilliefors, 1967] (an adaptation of the Kolmogorov-Smirnov test) to check the reliability of the fit. If the null hypothesis is rejected, the parameters are taken with caution and not registered.

\section{Analysis of the flag dynamics}

In the following, we focus on three indicators of chaos: (1) the power spectral densities, (2) the maximal Lyapunov exponents, and (3) the algorithm results (i.e. the statistical test of Lillierfors and the tail index). 

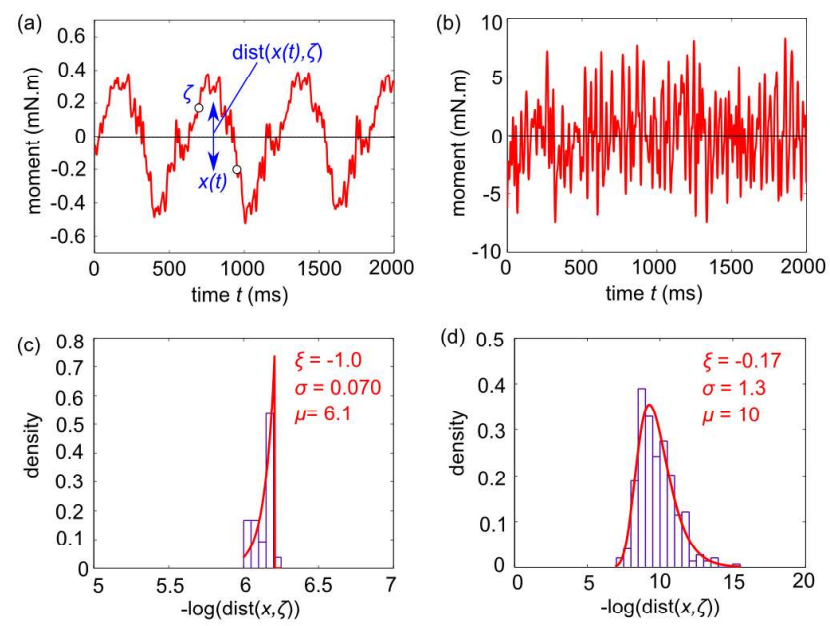

Fig. 2. (a) Time series of flapping moment at $U=0.9 \mathrm{~m} / \mathrm{s}$. The flapping is periodic with a noisy background. (b) The same at $U=13.8 \mathrm{~m} / \mathrm{s}$, without any clear periodicity. (c) Distribution of the maxima of $y(t)$ at $U=0.9 \mathrm{~m} / \mathrm{s}[\mathrm{Fig}$. $2(\mathrm{a})]$ with a random value $\zeta=0.1981$. The red curve is the best fit to eq. (1) with parameters indicated beside. In this situation the parameter $\xi$ is moved to the boundary of the parameter space $\xi \in[-1,1]$ and the fit is unsuccessful. (d) The same distribution at $U=13.8 \mathrm{~m} / \mathrm{s}$ [Fig. 2(b)] with a random value $\zeta=0.4562$.

The indicators are computed with the time series of flapping moment, by applying thirty-nine values of wind velocity to the flag.

As a rough indicator of chaos, the power spectral densities are reported in Fig. 3(a), where we observe a sharp frequency peak for $U \simeq 0.5-4 \mathrm{~m} / \mathrm{s}$, suggesting a strongly periodic flapping. The linear increase of the dominant frequency is consistent with previous experimental and numerical works (see e.g. [Virot et al., 2013] and references therein). Above $U \simeq 4 \mathrm{~m} / \mathrm{s}$, a continuous spectral enrichment is observed and a growing low-frequency domain appears at $U \simeq 9 \mathrm{~m} / \mathrm{s}$. Intuitively, these features are indicative of a transition towards chaotic behavior [Manneville, 2004].

Prior to the algorithm results, we discuss the maximum Lyapunov exponent, by following the steps detailed in [Wolf et al., 1985]. The computation implies the arbitrary choice of a time-delay for analyzing an equivalent attractor [Packard et al., 1980; Takens \& Mañé, 1981], and more particularly the knowledge of its embedding dimension. In order to do so we attempted two different methods [Cao, 1997; Gautama et al., 2003], but in both cases the embedding dimension remained out of reach: both methods rely on the minimization of a function for which we did not find reliable minima. Our observations lead us to hypothesize that it is linked to the short character of the time series. Actually, even if an embedding dimension were extracted, there is no way of stating whether the method has converged to a reliable value or if the time series is too short. As a result, the computation of the maximal Lyapunov exponents can be misleading, and does not clarify whether the flapping is chaotic or not in our case.

We therefore apply the algorithm based on recurring values to discriminate between chaotic and nonchaotic dynamics. The time series of flapping moment are sampled in $n=56$ blocks containing $m \simeq 260 \mathrm{~ms}$ (i.e. 250 observations). As a first step, the results of the statistical test of Lillierfors are presented in Fig. 3(b). A test parameter lower than the critical value 0.4 means that in average there is no differences between the observed distributions and the generalized extreme value distribution [eq. (1)] at the $10 \%$ significance level. If the test is not rejected, we consider that the data are reliable for the algorithm. We notice that when the flapping frequency is lower than $4 \mathrm{~Hz}$ (i.e. below $U=2 \mathrm{~m} / \mathrm{s}$ ), less than one period is included in a block and the Lillierfors test is rejected. As previously pointed out, the detection of chaos is based on the value of the tail index $\xi$ : if $\xi \simeq 0$, the fit to the Gumbel distribution succeeds and the dynamic is chaotic. Otherwise the dynamics is periodic, based on the analysis of recurrence distribution obtained. The underlying recurrence distribution for the periodic case is a Dirac's delta [Lucarini et al., 2016]. In the quasi-periodic case, the distribution is not bell-shaped but rather narrow as the one observed in Fig. 2(c). There is no other possibility for the non-convergence to the extreme value laws, but note that as studied in 


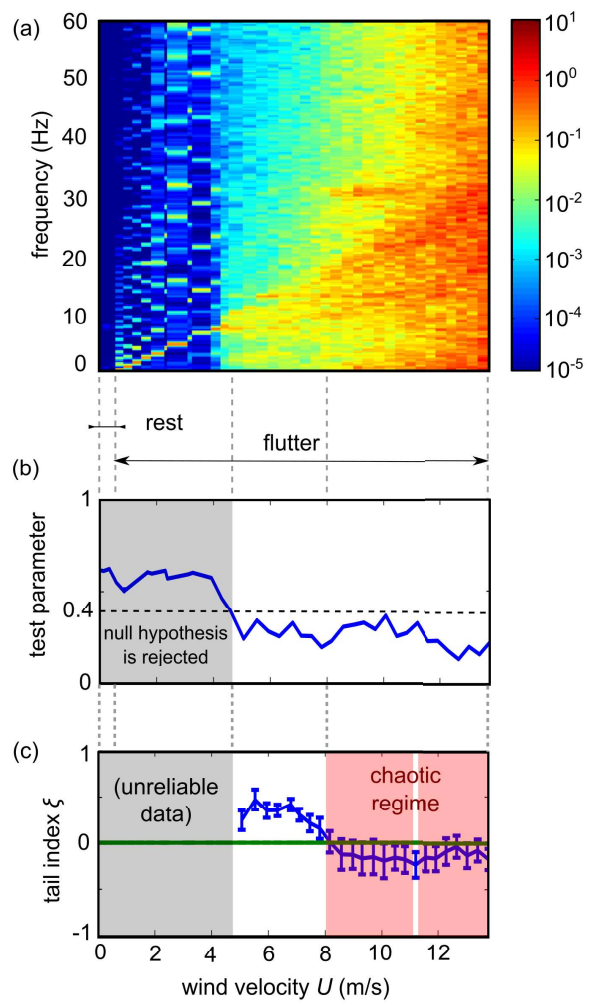

Fig. 3. (a) Evolution of the power spectral densities of the flapping moment when the wind velocity is increased. The flag starts to flutter at $U \simeq 0.5 \mathrm{~m} / \mathrm{s}$. (b) Algorithm results. The gray area indicates where the data are considered to be not reliable (data in this regime are not shown). (c) Tail index $\xi$. The red area indicates where the convergence towards a Gumbel distribution is achieved $(\xi \simeq 0)$ i.e. where the dynamic is chaotic. Error-bars correspond to the standard deviations of $\xi$ for hundred random values $\zeta$ in the algorithm.

[Faranda et al., 2013; Faranda \& Vaienti, 2013], strong noise can also bring the statistical convergence of the recurrences to the extreme value distributions. The evolution of the tail index $\xi$ is presented in Fig. 3(c). We observe a rather continuous transition from periodic flapping to chaotic flapping at $U \simeq 8 \mathrm{~m} / \mathrm{s}$. This threshold is consistent with the appearance of low frequencies in the power spectral densities [Fig. 3(a)]. Nevertheless, there is a systematic drift of $\xi$ towards negative values, and a short non-chaotic regime is detected at $U \simeq 11 \mathrm{~m} / \mathrm{s}$, mirroring the intrinsic sensibility of the algorithm to statistical fluctuations. The critical value of the test parameter fixed for the acceptance of the Lilliefors test is higher than the one prescribed for independent and identically distributed variables (i.e. approximately 0.2 for $n \simeq 50$ maxima) which is achieved only for wind velocity higher than $12 \mathrm{~m} / \mathrm{s}$. This value takes into account the dependence structure between the maxima. We found that maxima are dependent in clusters of average length 2 . This can be quantified by the extremal index [Freitas et al., 2012].

We can hypothesize three different behaviors: a first one for $U<4.5 \mathrm{~m} / \mathrm{s}$, where the maxima are dependent and the test parameter is higher than 0.4 ; a second one in the range $U=4.5-12 \mathrm{~m} / \mathrm{s}$, where the maxima are dependent but where the shape of their histogram approach the generalized extreme value distribution; and a third one for $U>12 \mathrm{~m} / \mathrm{s}$, where the maxima are almost independent and identically distributed and consistent with the generalized extreme value distribution according to the statistical test.

\section{Discussion and perspectives}

We have shown that the algorithm can provide information on the location of chaotic regimes. We stressed the fact that it is adapted to time series of limited size (as usually encountered) because it states its own convergence unlike classical tools. Let us now examine the limits of this algorithm by reporting its 


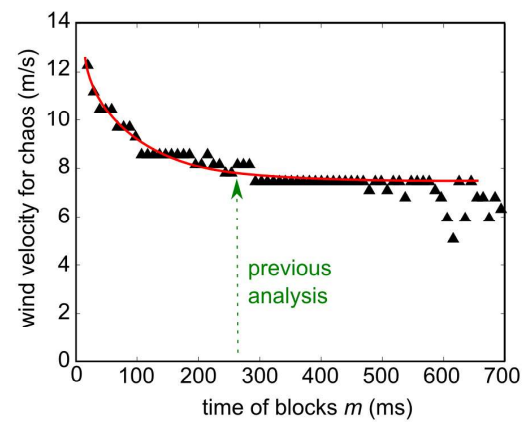

Fig. 4. Sensibility of the algorithm to the sampling. The minimal wind velocity required to detect chaotic flapping (determined when the tail index is zero) seem to converge towards $8 \mathrm{~m} / \mathrm{s}$, but with many discrepancies above 600 ms. The red curve is a guide to the eyes. The arrow indicates the block size chosen to do the previous analysis.

dependence to the sampling of the time series. When fixing the number of blocks $n$ or the number of observations $m$ in each block ( $\mathrm{nm}$ being the size of the time series), the time scale at which we observe the temporal recurrences in the algorithm get fixed. It therefore imposes the lowest frequency at which we can analyze the dynamic of the time series. In the case of Fig. 3(c), we have chosen $m \simeq 260 \mathrm{~ms}$, thus we "hide" the frequency range $0-4 \mathrm{~Hz}$. For judging the validity of the algorithm results, we report in Fig. 4 the values of the wind velocity required for chaos (determined when the tail index is zero) when the time scale of blocks is arbitrarily varied. We observe a decreasing trend below $100 \mathrm{~ms}$, i.e. when the hidden frequency range is of the order of the flapping frequency $(\sim 10 \mathrm{~Hz})$. On the other hand, when the time of observation is larger than $600 \mathrm{~ms}$, we see many discrepancies which can be associated to the fact that less than $n=25$ values are used for reconstructing the distributions of maxima, probably not enough.

We can conclude that this framework needs to be applied with care: if the time series is sampled in too many blocks the typical frequency of the system can be hidden, giving misleading results. Conversely, if too few blocks are chosen (typically less than 25 for this problem), the distributions can not be reconstructed properly, and many discrepancies are reported.

We have discussed a framework which bypasses a series of difficulties classically linked to the estimation of the embedding dimension. Such analyses could be a gateway for matching prediction of dynamical system theory together with natural systems.

\section{Acknowledgments}

We thank Paul Manneville and Olivier Cadot for their help in the early stages of the project. We are grateful to Benoît Roman for providing bi-oriented polypropylene sheets. E.V. was supported by the EDX-DGA program and D.F. was supported by the ERC Grant A2C2 (No. 338965).

\section{References}

Ait Abderrahmane, H., Païdoussis, M. P. \& Fayed, M. \& Ng, H. D. [2012], "Flapping dynamics of a flexible filament," Phys. Rev. E 84, 066604.

Ait Abderrahmane, H., Païdoussis, M. P., Fayed, M. \& Ng, H. D. [2012], "Nonlinear dynamics of silk and Mylar flags flapping in axial flow," J. Wind Eng. Ind. Aerod. 107-108, 225-236.

Alben, S. \& Shelley, M. J. [2008], "Flapping states of a flag in an inviscid fluid: bistability and the transition to chaos," Phys. Rev. Lett. 100, 074301.

Cao, L. [1997], "Practical method for determining the minimum embedding dimension of a scalar time series," Physica D 110, 43-50.

Connell, B.S.H. \& Yue, D. K. P. [2007], "Flapping dynamics of a flag in a uniform stream," J. Fluid Mech. 581, 33-67.

Collet, P. [2001], "Statistics of closest return for some non-uniformly hyperbolic systems," Ergod. Theor. Dyn. Syst. 21, 401-420. 
Faranda, D., Lucarini, V., Turchetti, G. \& Vaienti S. [2011], "Numerical convergence of the Block Maxima approach to the Generalized Extreme Value distribution," J. Stat. Phys. 145, 1156-1180.

Faranda, D., Lucarini, V., Turchetti, G. \& Vaienti, S, [2012], "Generalized extreme value distribution parameters as dynamical indicators of stability," Int. J. Bifurcat. Chaos 22, 1250276.

Faranda, D., Freitas, J. M., Lucarini, V., Turchetti, G. \& Vaienti, S. [2013], "Extreme value statistics for dynamical systems with noise," Nonlinearity 26, 2597.

Faranda, D. \& Vaienti, S. [2013], "A recurrence-based technique for detecting genuine extremes in instrumental temperature records." Geophys. Res. Lett. 40, 5782-5786.

Freitas, A. C. M. \& Freitas, J. M. [2008], "On the link between dependence and independence in extreme value theory for dynamical systems," Stat. Probabil. Lett. 78, 1088-1093.

Freitas, A. C. M., Freitas, J. M. \& Todd, M. [2009], "Hitting time statistics and extreme value theory," Probab. Theory Rel. 147, 675-710.

Freitas, A. C. M., Freitas, J. M., Todd, M. [2012], "The extremal index, hitting time statistics and periodicity," Advances in Mathematics 231, 2626-2665.

Gautama, T., Mandic, D. P. \& Van Hulle, M. M. [2003], "A differential entropy based method for determining the optmal embedding parameters of a signal," Proc. ICASSP.

Gleick, J. [1987], Chaos: making a new science, (Penguin Books, USA).

Gnedenko, B. [1943], "Sur la distribution limite du terme maximum d'une série aléatoire," Ann. Math. 44, 423-453.

Gupta, C., Holland, M. \& Nicol, M. [2011], "Extreme value theory for dispersing billiards and a class of hyperbolic maps with singularities," Ergod. Theor. Dyn. Syst. 31:1363-1390.

Huang, W. X. \& Sung, H. J. [2010], "Three-dimensional simulation of flapping flag in a uniform flow," $J$. Fluid Mech. 653, 301-336.

Leadbetter, M. R., Lindgren \& G., Rootzn, H. [1982], Extremes and related properties of random sequences and processes, (Springer, USA).

Lilliefors, H. W. [1967], "On the Kolmogorov-Smirnov test for normality with mean and variance unknown," J. Am. Stat. Assoc. 62, 399-402.

Lucarini, V., Faranda, D., Turchetti, G. \& Vaienti, S. [2012], "Extreme value theory for singular measures," Chaos 22, 023135.

Lucarini, V. et al. [2016], Extremes and Recurrence in Dynamical Systems, (Wiley, Hoboken).

Manneville, P. [2004], Instabilités, chaos et turbulence, (Éditions de l'École Polytechnique, France), p. 82.

Michelin, S., Llewellyn Smith, S. G. \& Glover, B.J. [2008], "Vortex shedding model of a flapping flag," J. Fluid Mech. 617, 1-10.

Packard, N. H., Crutchfield, J. P. Farmer, J. D. \& Shaw, R. S. [1980], "Geometry from a time series," Phys. Rev. Lett. 45, 712-716.

Poincaré, H. [1890], "Sur le problème des trois corps et les équations de la dynamique," Acta Math. 13, 3-270.

Shelley, M. J. \& Zhang, J. [2011], "Flapping and bending bodies interacting with fluid flows," Annu. Rev. Fluid Mech. 43, 449-465.

Takens, F. \& Mañé, R. [1981], Dynamical systems and turbulence, In: Rand, R., Young, L.S., Warwick Notes in Mathematics, (Springer, USA).

Taneda, S. [1968], "Waving motions of flags," J. Phys. Soc. Japan 24, 392-401.

Yadykin, Y., Tenetov, V. \& Levin, D. [2001], "The flow-induced vibration of a flexible strip hanging vertically in a parallel flow. Part 1: temporal aeroelastic instability," J. Fluids Struct. 15, 1167-1185.

Virot E., Amandolese X. \& Hémon P. [2013], "Fluttering flags: an experimental study of unsteady forces," J. Fluids Struct. 43, 385-401.

Virot E. [2015], Flottement de drapeau: dynamique et couplage (Ph.D. Thesis, École Polytechnique), 123126.

Virot E., Amandolese X. \& Hémon P. [2016], "Coupling between a flag and a spring-mass oscillator," J. Fluids Struct. 65, 447-454.

Wolf, A., Swift, J. B., Swinney, H. L. \& Vastano, J. A. [1985], "Determining Lyapunov exponents from a time series," Physica D 16, 285-317. 
Zhao, W., Païdoussis, M. P., Tang, L., Liu, M. \& Jiang, J. [2012], "Theoretical and experimental investigations of the dynamics of cantilevered flexible plates subjected to axial flow," J. Sound Vib. 331, $575-587$.

Xia Y., Michelin S. \& Doaré O. [2015], "Resonance-induced enhancement of the energy harvesting performance of piezoelectric flags," Appl. Phys. Lett. 107, 263901. 